\title{
ON THE GOLDIE DIMENSION OF INJECTIVE MODULES*
}

\author{
by JOSÉ L. GÓMEZ PARDO and PEDRO A. GUIL ASENSIO
}

(Received 23rd May 1996)

\begin{abstract}
Let $M$ be an essentially finitely generated injective (or, more generally, quasi-continuous) module. It is shown that if $M$ satisfies a mild uniqueness condition on essential closures of certain submodules, then the existence of an infinite independent set of submodules of $M$ implies the existence of a larger independent set on some quotient of $M$ modulo a directed union of direct summands. This provides new characterisations of injective (or quasi-continuous) modules of finite Goldie dimension. These results are then applied to the study of indecomposable decompositions of quasi-continuous modules and nonsingular CS modules.
\end{abstract}

1991 Mathematics subject classification: Primary 16P60; Secondary 16D50.

\section{Introduction}

It is well known that a finite-dimensional module $M$ can have quotient modules with infinite Goldie dimension. However, if $N$ is a directed union of direct summands of $M$ (we then say that $N$ is a directly split submodule of $M$ ), then $N$ itself is a direct summand of $M$ and, in particular, we have for the Goldie dimensions that $G d(M / N) \leq G d(M)$ (see[6]).

In this paper we analyse the significance of this condition for the finite-dimensionality of $M$. This is a departure from the more standard process of looking directly at submodules of $M$ and, somewhat paradoxically, it requires to take into account modules of infinite Goldie dimension. Thus we recall from [2] that $G d(M)$ is defined, in general, as the supremum of all cardinals $\kappa$ such that $M$ contains the direct sum of $\kappa$ nonzero submodules. It is clear that our condition is not sufficient for finite-dimensionality (consider, for example, a non-finitely generated semisimple module), so that we will also assume that $M$ is essentially finitely generated - that is, it contains an essential finitely generated submodule. Of course, the latter condition is satisfied by all finite-dimensional modules, and it will be also convenient to assume that $M$ is injective or, at least, that it satisfies a weaker injectivity condition such as being CS or quasi-continuous. This is not unreasonable in view of the fact that if we start with an essentially finitely generated module $M$, then its injective envelope is also essentially finitely generated and has the same Goldie dimension as $M$.

Our main result shows that if $M$ is an essentially finitely generated injective (or, more generally, quasi-continuous) module that satisfies a mild uniqueness condition on

\footnotetext{
* Work partially supported by the DGICYT (PB93-0515, Spain).
} 
essential closures of certain submodules, then the existence of an independent set of infinite cardinality $\$$ of nonzero submodules forces the existence of a larger independent set of nonzero submodules in some quotient $M / N$, where $N$ is a directly split submodule of $M$. There is, however, a technical difficulty that prevents a straightforward translation of the result in terms of the Goldie dimension of the involved modules, and this is the fact that $G d(M)=\kappa$ does not imply that $M$ contains a direct sum of $N$ nonzero submodules. When this happens, we say as in [2] that $G d(M)$ is attained in $M$. Thus our main result can be applied to a module $M$ such that $G d(M)=N$ is infinite and attained in $M$ and, under the aforementioned hypotheses, gives the existence of a directly split submodule $N$ such that $G d(M / N)>G d(M)$.

We stress that the assumption that $G d(M)$ is attained is not a very restrictive hypothesis. To put this in perspective, recall that an infinite cardinal $\kappa$ is called regular if $\kappa_{i}<\kappa$ for $i \in I$ with $|I|<\kappa$ implies $\Sigma \kappa_{i}<\kappa$ (where $|I|$ denotes the cardinality if $I$ ). An uncountable, regular, limit cardinal is said to be (weakly) inaccessible [9]. Now, the main result of [2] is that if $G d(M)$ is not an inaccessible cardinal, then $G d(M)$ is attained. Inaccessible cardinals are rather scarce if they exist at all: it is well known that their existence cannot be proved in ZFC (Zermelo-Fraenkel set theory with the Axiom of Choice), not even with the Generalised Continuum Hypothesis (GCH) thrown in.

However, if one is not ready to take for granted the nonexistence of inaccessible cardinals, it is necessary to bear in mind the possibility that $G d(M)$ is not attained. The reason is that, as explained below, it can be deduced from a classical result of Erdös and Tarski [5] on Boolean algebras that, for each inaccessible cardinal $\kappa$, there exists a self-injective ring $R$ such that $G d(R)=\kappa$ is not attained in $R$.

Even though our results are stated, more generally, for quasi-continuous or CS modules, most of them are new even in case $M$ is injective. Our main result is formulated in terms of infinite cardinals, but we obtain from it useful characterisations of finite-dimensional modules. In particular, we show that if $M$ is either quasicontinuous or a nonsingular CS module, then $M$ is finite-dimensional if and only if it is essentially finitely generated and every directly split quotient has countable Goldie dimension. Observe that $|M|$ is an obvious upper bound for $G d(M)$. In a variety of situations we obtain (directly split) submodules $N$ of $M$ such that $G d(M)<G d(M / N)$. In these cases it is clear that $G d(M)<|M|$ and, in particular, that the countable modules which satisfy the required hypotheses are finite-dimensional. This allows us to show that every countable quasi-continuous module is a direct sum of uniform modules, thus extending [3, Theorem 15], where the analogous result was proved for countable continuous modules. Similarly, we prove that every countable module $M$ such that $M$ is nonsingular (or, more generally, a UC-module in the sense of [13]) and every closed submodule is essentially finitely generated, is finite-dimensional. We also give some other applications and, in particular, we obtain a new characterisation of right artinian rings with Morita duality in terms of the right minimal injective cogenerator.

The proof of our main result relies on a counting argument based on "Tarski's lemma" [15], which had been applied by Osofsky in [11] (see also $[7,8,12]$ ) to deduce 
the finiteness of the socle of an injective cogenerator ring. When the Goldie dimension of $M$ is a regular infinite cardinal we show in the last part of the paper that the preceding results can be strengthened. In this case we prove a variant of Tarski's lemma that allows us to relax the assumptions of our main result.

Throughout this paper all rings $R$ will be associative and with identity, and Mod- $R$ will denote the category of right $R$-modules. By a module we will usually mean a right $R$-module. We refer to $[9,14,16]$ for all undefined notions used in the text.

\section{Finite-dimensional CS modules}

Recall that a submodule $K$ of an $R$-module $M$ is said to be closed (in $M$ ) when $K$ has no proper essential extensions in $M$. If $L \subseteq M$, then a closed submodule of $K$ of $M$ that contains $L$ as an essential submodule (we then write $L \subseteq_{e} K$ ) is called an essential closure of $L$ in $M$. The module $M$ is called CS (or an extending module, cf. [4]), if every closed submodule is a direct summand. $M$ is quasi-continuous (or $\pi$-injective, cf. [4]) if it is CS and whenever $M_{1}$ and $M_{2}$ are direct summands of $M$ with $M_{1} \cap M_{2}=0$ then $M_{1} \oplus M_{2}$ is also a direct summand of $M$.

A submodule $N \subseteq M$ will be called a directly split submodule when $N$ is a directed union of direct summands of $M$. A set $\left\{N_{j} \mid j \in J\right\}$ of independent submodules of $M$ is called a local direct summand of $M$ (cf. [4, p. 66]) when $\Sigma_{j \in F} N_{j}$ is a direct summand of $M$ for every finite subset $F \subseteq J$. If, furthermore, $|J|<\aleph$, then we will also say that the submodule $N=\oplus_{j \in J} N_{j}$ of $M$ is an $\kappa^{<}$-local direct summand. Observe that a local direct summand is, in particular, a directly split submodule.

The successor cardinal of a cardinal $\kappa$ will be denoted by $\kappa^{+}$. We say that the cardinal $\mathcal{N}$ is attained in $M$ when $M$ contains a direct sum of $\aleph$ nonzero submodules. If $\aleph$ is an infinite cardinal, we say that $M$ is $\aleph$-generated if it has a generating set $X$ such that $|X| \leq \aleph$. For later use we will also recall Tarski's lemma ([15, Théorème 7]):

Lemma 2.1. Let $\kappa, \alpha, \beta$ be cardinal numbers such that $\kappa$ is infinite and $\alpha, \beta>1$. Suppose that $\alpha$ is the smallest cardinal such that $N<\beta^{\alpha}$. Then each set of cardinality $\kappa$ can be decomposed into a class of subsets $\mathcal{K}$ with $|\mathcal{K}|=\beta^{x}$ and such that $|X|=\alpha$ for each $X \in \mathcal{K}$, and $|X \cap Y|<\alpha$ for $X, Y \in \mathcal{K}, X \neq Y$.

We now give our main result.

Theorem 2.2. Let $R$ be a ring and $M$ an essentially finitely generated quasi-continuous right $R$-module. Suppose that $N$ is an infinite cardinal such that $N$ is attained in $M$ and every $N^{<-l o c a l ~ d i r e c t ~ s u m m a n d ~ o f ~} M$ has a unique (as a set) essential closure in $M$. Then there exists a directly split submodule $N$ of $M$ such that $\mathrm{N}^{+}$is attained in $M / N$.

Proof. For each submodule $K$ of $M$, denote by $E(K)$ an essential closure in $M$ of $K$, so that $E(K)$ is a direct summand of $M$. Let $\left\{C_{i}\right\}_{i \in I}$ be an independent set of nonzero submodules of $M$ such that $|I|=\kappa$. Choose, for each $i \in I$, a fixed essential closure $E_{i}$ 
of $C_{i}$ in $M$ and, for each subset $A$ of $I$, a (not necessarily unique) essential closure $E_{A}=E\left(\oplus_{i \in A} E_{i}\right)=E\left(\oplus_{i \in A} C_{i}\right) \subseteq M$. Since $I$ is infinite, it follows from Tarski's lemma that there exists a set $\mathcal{K} \subseteq 2^{l}$ and an infinite cardinal $\kappa \leq \kappa$ such that $|\mathcal{K}|>\kappa,|X|=\kappa$ for each $X \in \mathcal{K}$, and $|X \cap Y|<\kappa$ for all $X, Y \in \mathcal{K}$ such that $X \neq Y$. Let now $\mathcal{N}=\{A \subseteq I \| A \mid<\kappa\}$ and define $N:=\Sigma_{A \in \mathcal{N}} E_{A} \subseteq M$. Because $M$ is quasi-continuous each (direct) sum of an independent set of direct summands of $M$ is a local direct summand and so, for each $A \in \mathcal{N}, E_{A}$ is the unique essential closure in $M$ of the $\mathcal{K}^{<-}$ local direct summand $\oplus_{i \in A} E_{i}$. Thus if $A \in \mathcal{N}, A \subseteq B \subseteq I$, we have that $E_{A} \subseteq E_{B}$ and so $N$ is a directed union of direct summands of $M$ and hence a directly split submodule.

We claim that $\left\{\left(E_{X}+N\right) / N\right\}_{X \in \mathcal{K}}$ is an independent set of nonzero submodules of $M / N$. First we show that, for each $X \in \mathcal{K},\left(E_{X}+N\right) / N \neq 0$. Assume on the contrary that $E_{X} \subseteq N$ for some $X \in \mathcal{K}$. Since $M$ is essentially finitely generated, so is the direct summand $E_{X}$, and hence it contains a finitely generated essential submodule $L_{X}$. Then $L_{X} \subseteq E_{X} \subseteq N=\Sigma_{A \in \mathcal{N}} E_{A}$ and so there exists a finite set $J \subseteq \mathcal{N}$ such that $L_{X} \subseteq \Sigma_{A \in J} E_{A}$. Since $L_{X}$ is essential in $E_{X}, \quad\left\{L_{X} \cap E_{i}\right\}_{i \in X}$ is an independent family of nonzero submodules of $L_{X}$ and, for each $i \in X$ we have that

$$
0 \neq L_{X} \cap E_{i} \subseteq L_{X} \subseteq \Sigma_{A \in J} E_{A} \subseteq E_{\cup_{A \in J} A}
$$

where the last inclusion is a consequence of the fact that for each $A \in J$, the unique essential closure $E_{A}$ of the sum $\oplus_{i \in A} E_{i}$ is contained in $E_{\mathrm{U}_{A \in} A}$. On the other hand, since $J$ is finite and $|A|<\kappa$ for each $A \in J$, we have that $\left|\cup_{A \in J} A\right|<\kappa$. Then we obtain

$$
\left|\cup_{A \in J} A\right|<\kappa=|X|
$$

and so there exists $j \in X$ such that $j \notin \cup_{A \in J} A$. Thus we have that $E_{j} \cap\left(\oplus_{\cup_{A \in J} A} E_{i}\right)=0$ and hence $E_{j} \cap E_{\cup_{A \in J A}}=0$, which gives a contradiction and shows that $E_{X}$ is not contained in $N$.

Finally, in order to show that the set $\left\{\left(E_{X}+N\right) / N\right\}_{X \in \mathcal{K}}$ is independent, we have to prove that for each finite set $\left\{Y_{1}, \ldots, Y_{r}\right\}$ with $Y_{k} \in \mathcal{K}$ and $Y_{k} \neq X, k=1, \ldots, r$, we have that $E_{X} \cap\left(\Sigma_{k=1}^{r} E_{Y_{k}}\right) \subseteq N$. For each $k \in\{1, \ldots, r\}$, let $Y^{k}:=Y_{k} \cap\left(\cup_{j=1}^{k-1} Y_{j}\right)$. Then we can write $Y_{k}=\left(Y_{k}-Y^{k}\right) \cup Y^{k}$, where the union is disjoint and so $\oplus_{Y_{k}} E_{i}=\left(\oplus_{Y_{k}-Y^{k}} E_{i}\right) \oplus\left(\oplus_{Y^{k}} E_{i}\right)$. Since $M$ is a quasi-continuous module, it is clear that $E_{Y_{k}}=E\left(\oplus_{Y_{k}} E_{i}\right)=E\left(\oplus_{Y_{k}-Y_{k}} E_{i}\right) \oplus E\left(\oplus_{\gamma^{k}} E_{i}\right)$ and, because $\left|Y^{k}\right|=\left|\cup_{j=1}^{k-1}\left(Y_{k} \cap Y_{j}\right)\right| \leq \Sigma_{j=1}^{k-1}\left|Y_{k} \cap Y_{j}\right|<\kappa \leq \kappa$, we have that $E\left(\oplus_{\gamma^{k}} E_{i}\right)=E_{\gamma^{k}}$, the unique essential closure of $\oplus_{Y^{k}} E_{i}$ in $M$. Moreover, $Y^{k}$ can be written as a disjoint union of subsets of $Y_{1}, Y_{2}, \ldots, Y_{k-1}$, each of which has cardinality $<\kappa$ and hence we see that $E_{Y^{k}} \subseteq \Sigma_{j=1}^{k-1} E_{Y_{j}}$. Then it follows that $\Sigma_{k=1}^{r} E_{Y_{k}}=\Sigma_{k=1}^{r} E\left(\oplus_{Y_{k}-Y^{k}} E_{i}\right)$. Since $\cup_{k=1}^{r} Y_{k}=$ $\cup_{k=1}^{r}\left(Y_{k}-Y^{k}\right)$, where the last union is disjoint, we have by the quasi-continuity of $M$ that $\Sigma_{k=1}^{r} E_{Y_{k}}=\oplus_{k=1}^{\prime} E\left(\oplus_{Y_{k}-\gamma^{k}} E_{i}\right)=E\left(\oplus_{\cup_{k=1}^{r} Y_{k}} E_{i}\right)$ is an essential closure of $\oplus_{\cup_{k=1}^{r} \gamma_{k}} E_{i}$ and so we see that $\oplus_{U_{k=1}^{\prime} Y_{k}} E_{i} \subseteq_{e} \Sigma_{k=1}^{r} E_{Y_{k}}$.

Now, it is clear that $\left(\oplus_{X} E_{i}\right) \cap\left(\oplus_{u_{k=1}^{r}} Y_{k} E_{i}\right)$ is an essential submodule of $E_{X} \cap\left(\Sigma_{k=1}^{r} E_{Y_{k}}\right)$ and, furthermore, $\left(\oplus_{X} E_{i}\right) \cap\left(\oplus_{\cup_{k=1}^{r}} Y_{k} E_{i}\right)=\oplus_{H} E_{i}$, where $H=X \cap\left(\cup_{k=1}^{r} Y_{k}\right)$. Therefore, $\oplus_{H} E_{i}$ is an essential submodule of $E_{X} \cap\left(\Sigma_{k=1}^{r} E_{Y_{k}}\right)$. Since $|H|=\left|X \cap\left(\cup_{k=1}^{r} Y_{k}\right)\right| \leq$ $\Sigma_{k=1}^{r}\left|X \cap Y_{k}\right|<\kappa$, we see that $E_{H}$ is the unique essential closure of $\oplus_{H} E_{i}$ in $M$ and so $E_{X} \cap\left(\Sigma_{k=1}^{r} E_{Y_{k}}\right) \subseteq E_{H}$. But $H \in \mathcal{N}$ and hence $E_{H} \subseteq N$, which completes the proof. 
Erdös and Tarski [5, Corollary 2] constructed, for any inaccessible cardinal $\kappa$, a complete boolean algebra $B$ such that the supremum of the sizes of sets consisting of pairwise disjoint elements of $B$ is $\kappa$ but $B$ does not contain $\kappa$ pairwise disjoint elements. If $B$ is regarded as a boolean ring, then $B$ is self-injective [14, Corollary XII.3.5] and $G d(B)=\kappa$ is not attained in $B$. For this reason we include the hypothesis that $G d(M)$ is attained in the next corollary, which is essentially a reformulation of Theorem 2.2 in terms of Goldie dimension.

Corollary 2.3. Let $R$ be a ring and $M$ a quasi-continuous right $R$-module. Then $M$ is finite dimensional if and only if the following conditions hold:

(i) Every local direct summand of $M$ has a unique essential closure in $M$.

(ii) $M$ is essentially finitely generated.

(iii) $G d(M)$ is attained in $M$ and for every directly split submodule $N$ of $M$, $G d(M / N) \leq G d(M)$.

Proof. If $M$ is finite dimensional, then every local direct summand is clearly a direct summand and hence (i) holds. Furthermore, $M$ is essentially finitely generated, so that (ii) is satisfied. Finally, $M$ has ACC on direct summands ([6]), so that every directly split quotient of $M$ is actually a direct summand and (iii) also holds.

Conversely, assume that conditions (i)-(iii) hold but $M$ is not finite dimensional and let $G d(M)=\aleph \geq \aleph_{0}$. Then by Theorem 2.2 there exists a directly split submodule $N \subseteq M$ such that $G d(M / N)>\aleph$, which gives a contradiction and completes the proof.

There is an important special case in which the hypothesis that each $\aleph^{<}$-local direct summand of the quasi-continuous module $M$ has a unique essential closure is automatically satisfied, namely, when $\kappa=\kappa_{0}$. Then every $\kappa^{<-l o c a l}$ direct summand of $M$ is a direct summand, so that we have:

Corollary 2.4. Let $R$ be $a$ ring and $M$ a quasi-continuous right $R$-module. Then the following assertions hold:

(i) $M$ is finite-dimensional if and only if $M$ is essentially finitely generated and every directly split quotient of $M$ has countable Goldie dimension.

(ii) $M$ is finitely cogenerated if and only if it is essentially finitely generated and every directly split quotient of $M$ has countably generated essential socle.

Proof. (i) The necessity is clear. For the sufficiency, observe that if $G d(M)$ is countable but $M$ is not finite-dimensional, then $G d(M)=\aleph_{0}$ is attained as $\kappa_{0}$ is not inaccessible, and so the result follows from Theorem 2.2 bearing in mind the preceding remarks.

(ii) follows from (i). 
In relation with the preceding corollary, observe that each directly split quotient of the $\mathbb{Z}$-module $\mathbb{Q} / \mathbb{Z}$ is a direct summand and hence has countable Goldie dimension (and countably generated essential socle), so that the hypothesis that $M$ is essentially finitely generated cannot be removed. On the other hand, let $R=\Pi_{l} k_{i}$ be a denumerable product of fields (or skew-fields) and $M=R_{R}=E\left(\oplus_{l} k_{i}\right.$ ) where $\oplus_{1} k_{i}=\operatorname{Soc}\left(R_{R}\right)$. Then $M$ is finitely generated and $G d(M)=\aleph_{0}$, so that the hypothesis that every directly split quotient of $M$ has countable Goldie dimension cannot be removed from Corollary 2.4. Similarly, neither of conditions (ii) and (iii) can be removed from Corollary 2.3 .

The next result extends [3, Theorem 15] (see also [4, 10.12]) from continuous to quasi-continuous modules.

Corollary 2.5. Any countable quasi-continuous module is a direct sum of uniform modules.

Proof. By the proof of [4, Lemma 10.1], $M$ is a direct sum of essentially finitely generated modules, which are finite-dimensional by Corollary 2.4 . Now the result follows from the fact that a finite-dimensional CS module is a finite direct sum of indecomposable modules, which are uniform.

As another consequence of the preceding results, we can give a characterisation of right artinian rings with Morita duality.

Corollary 2.6. Let $R$ be a ring. Then the following conditions are equivalent:

(i) $R$ is right artinian with a right Morita duality.

(ii) There exists a finitely generated injective right $R$-module $E$ with essential socle, such that each local direct summand of $E$ has a unique injective envelope in $E$ and every finitely generated right $R$-module embeds in a direct sum of copies of E.

(iii) There exists a finitely generated injective right $R$-module $E$ such that $\operatorname{Soc}(E)$ is essential and countably generated, and every finitely generated right $R$-module embeds in a direct sum of copies of $E$.

Proof. If $R$ is right artinian with Morita duality, it is enough to take $E_{R}$ equal to the minimal injective cogenerator which, obviously, satisfies all the conditions in (ii) and (iii). Conversely, assume that $E$ is a module as in (ii). Since $G d(E)$ is attained in $E$ by [2, Lemma 4], $E$ is finitely cogenerated by Corollary 2.3 and hence so is every finitely generated right $R$-module, so that $R$ is right artinian. Moreover, it is clear that $E$ is a cogenerator and so the minimal injective cogenerator of Mod- $R$ is a direct summand of $E$ and hence finitely generated. That (iii) implies (i) can be obtained in a similar way, using Corollary 2.4 . 
The preceding corollary can be obviously modified by requiring in condition (ii) only the weaker hypothesis that every $\kappa^{<}$-local direct summand of $E$ has a unique injective envelope in $E$, where $N$ is an infinite cardinal such that $\operatorname{Soc}(E)$ is $\aleph$-generated. Observe also that it is enough to assume that $E$ is quasi-continuous (instead of injective) and, on the opposite extreme, it can be supposed that $E$ is the minimal injective cogenerator.

If we do not assume that $M$ is quasi-continuous, we can still prove a result similar to Theorem 2.2 using a stronger uniqueness hypothesis of essential closures. We recall from [13] that $M$ is called a UC-module if each submodule has a unique essential closure. On the other hand, $M$ is said to be a CEF-module ([4]) if every closed submodule is essentially finitely generated. If $M$ is a UC-module, then the assignment $L \mapsto E(L)$ is order-preserving and defines a closure operator (see [14, Chapter III, §7] for the definition) in the lattice of submodules of $M$. Suppose that $\left\{C_{i}\right\}_{I}$ is an independent set of nonzero submodules of the UC-module $M$ with $E_{i}=E\left(C_{i}\right)$. Since the closure operator preserves intersections, $\left\{E_{i}\right\}_{l}$ is also an independent set and if $Y_{1}, \ldots, Y_{r}$ are subsets of $I$ it is not difficult to show that $\oplus_{\cup_{k=1}^{r}} Y_{k} E_{i} \subseteq_{e} \Sigma_{k=1}^{r} E_{Y_{k}}$, where $E_{Y_{k}}=E\left(\oplus_{Y_{k}} E_{i}\right)$. Thus we see that the UC property can replace the quasi-continuity in the last part of the proof of Theorem 2.2 and the argument used there goes through giving the following result:

Proposition 2.7. Suppose that $M$ is both a UC-module and a CEF-module. If $G d(M)$ is infinite and attained in $M$, then there exists a submodule $N$ of $M$ such that $N$ is a directed union of closed submodules of $M$ and $G d(M / N)>G d(M)$.

We remark that there exist finitely generated injective modules of denumerable Goldie dimension (cf. [12, p. 263]) which are not UC-modules. These modules satisfy the hypotheses of Theorem 2.2 and, in particular, we see that the uniqueness assumption of Theorem 2.2 is substantially weaker than $M$ being UC. However, we do not know in general whether it is possible to remove this uniqueness hypothesis from Theorem 2.2 and subsequent corollaries.

An important class of UC-modules is that of nonsingular modules (see, e.g. [13]). As an immediate consequence of the preceding proposition we have:

Corollary 2.8. If $M$ is a nonsingular $C E F$-module such that $G d(M)$ is attained in $M$, then $G d(M)<|M|$. In particular, if $M$ is a countable nonsingular module, then $M$ is finite-dimensional if and only if it is a CEF-module.

If we assume that the module $M$ is, furthermore, a CS module, then the CEF condition reduces to $M$ being essentially finitely generated and we have:

Corollary 2.9. Let $M$ be a nonsingular $C S$ right $R$-module. Then $M$ is finitedimensional if and only if $M$ is essentially finitely generated, $G d(M)$ is attained in $M$, and $G d(M / N) \leq G d(M)$ for every directly split submodule $N$ of $M$. In particular, any countable nonsingular CS module is a direct sum of uniform modules, and any countable right nonsingular right $C S$ ring has a semisimple maximal right quotient ring. 
The CS hypothesis cannot be dropped from Corollary 2.9. Let $k$ be a countable field, $k_{n}=k$ for each $n \in \mathbb{N}$ and $Q=\Pi_{\mathbb{N}} k_{n}$. Then the subring $R=k \cdot 1+\oplus_{\mathbb{N}} k_{n}$ of $Q$, consisting of the eventually constant sequences of elements of $k$ is a countable Von Neumann regular ring which is not finite-dimensional. In fact, the ideal $L=\left\{\left(x_{n}\right)_{\mathrm{N}} \in R \mid x_{n}=0\right.$ for $n$ even $\}$ is closed but not essentially finitely generated, so that this ring also shows that in Corollary 2.8 the hypothesis that $M$ is a CEF-module cannot be replaced by the weaker assumption that $M$ itself is essentially finitely generated.

Recall that a family of modules $\left\{M_{i} \mid i \in I\right\}$ is locally semi-T-nilpotent if, for any countable set of non-isomorphisms $\left\{f_{n}: M_{i_{n}} \rightarrow M_{i_{n+1}}\right\}$ with all $i_{n}$ distinct in $I$, and for any $x \in M_{i}$, there exists $k$ (depending on $x$ ) such that $f_{k} \ldots f_{1}(x)=0([10])$.

Corollary 2.10. Let $M$ be a countable module which is either quasi-continuous or nonsingular and CS. Then $M$ has a decomposition $M=\oplus_{l} M_{i}$ with $\left\{M_{i}\right\}_{l}$ locally semi-Tnilpotent.

Proof. If $M$ is quasi-continuous, then $M$ has an indecomposable decomposition $M=\oplus_{1} m_{i}$ by Corollary 2.5. Then it follows from [10, Theorem 2.22] that this decomposition complements direct summands and hence the family $\left\{M_{i}\right\}_{I}$ is locally semi-T-nilpotent by [10, Theorem 2.26].

If $M$ is nonsingular and CS then $M$ has an indecomposable decomposition $M=\oplus_{I} M_{i}$ by Corollary 2.9. Then $\left\{M_{i}\right\}_{l}$ is, again, semi-T-nilpotent by [1, Theorem 1$]$.

\section{Additional results for regular cardinals}

We will now show that the results obtained in the preceding section can be strengthened in the case that $G d(M)=\aleph$ with $\aleph$ a regular infinite cardinal. We shall need a variation on Tarski's lemma. We assume, as usual, that the axiom of choice (AC) holds and hence every set can be well-ordered and we can view a cardinal number as an initial ordinal (cf. [9, Theorem 9.1.5]). Thus every infinite cardinal $\chi$ is a limit ordinal and its cofinality $c f(\chi)$ is defined as the least ordinal number $\theta$ such that $\chi$ is the limit of an increasing sequence of smaller ordinals of length $\theta([9$, Definition 10.3.6]). Then $c f(\chi)$ is a limit ordinal such that $c f(\chi) \leq \chi$ and $\chi$ is regular when $c f(\chi)=\chi$. Since $c f(c f(\chi))=c f(\chi)$ for each infinite cardinal $\chi, c f(\chi)$ is a regular cardinal.

The following lemma can be obtained from Tarski's lemma, without using the regularity hypothesis but assuming instead that $\mathrm{GCH}$ holds (given $I$ with $|I|=\kappa$ infinite, just take $\beta=2$ in Lemma 2.1; see also Lemma 3.3 below). For regular cardinals (and we need regularity in order to be able to apply the argument of Theorem 2.2, cf. the proof of Theorem 3.2 below), we give a more direct proof that does not require $\mathrm{GCH}$.

Lemma 3.1. Let 1 be an infinite set such that $|I|=N$ is a regular cardinal. Then there exists a set $\mathcal{K} \subseteq 2^{I}$ such that $|\mathcal{K}|>\mathcal{N},|X|=\aleph$ for each $X \in \mathcal{K}$ and $|X \cap Y|<\mathcal{N}$ for $X, Y \in \mathcal{K}, X \neq Y$. 
Proof. Write $I=\cup_{A \in A} A$ as a union of pairwise disjoint subsets $A$ such that $|A|=\aleph$ for each $A \in \mathcal{A}$ and $|\mathcal{A}|=\aleph$. Using Zorn's lemma we obtain that there exists a subset $\mathcal{K}$ of $2^{I}$ which is maximal with respect to the following properties: (i) $\mathcal{A} \subseteq \mathcal{K}$, (ii) $|X|=\mathcal{N}$ for each $X \in \mathcal{K}$, and (iii) $|X \cap Y|<\mathcal{N}$ for each $X, Y \in \mathcal{K}$ such that $X \neq Y$. It suffices to prove that $|\mathcal{K}|>\mathcal{N}$. Well-order $I$ and write $I=\left\{i_{x} \mid \alpha<\mathcal{N}\right\}$, where $\alpha$ ranges over all ordinals strictly smaller than $\mathcal{N}$. Assume that $|\mathcal{K}|=|I|$ and let $f: I \rightarrow \mathcal{K}$ be a bijection. We claim that, for each ordinal $\alpha<\kappa, f\left(i_{\alpha}\right)$ is not contained in $\cup_{\beta<\alpha} f\left(i_{\beta}\right)$. Indeed, if $f\left(i_{\alpha}\right) \subseteq \cup_{\beta<\alpha} f\left(i_{\beta}\right)$, then $f\left(i_{\alpha}\right)=f\left(i_{\alpha}\right) \cap\left(\cup_{\beta<\alpha} f\left(i_{\beta}\right)\right)=\cup_{\beta<\alpha}\left(f\left(i_{\alpha}\right) \cap f\left(i_{\beta}\right)\right)$, so that

$$
\aleph=\left|f\left(i_{\alpha}\right)\right| \leq \Sigma_{\beta<\alpha}\left|f\left(i_{\alpha}\right) \cap f\left(i_{\beta}\right)\right|
$$

where each $\left|f\left(i_{\alpha}\right) \cap f\left(i_{\beta}\right)\right|<\aleph$ because $f$ is injective. Since $\alpha<\aleph$ and $\aleph$ is a regular cardinal, we obtain that $\left|f\left(i_{z}\right)\right|<\kappa$, which gives a contradiction and establishes our claim.

Consider now a subset $Z$ of $I$ obtained by choosing, for each $\alpha<\kappa$, an element $j_{\alpha} \in f\left(i_{\alpha}\right)$ such that $j_{\alpha} \notin \cup_{\beta<\alpha} f\left(i_{\beta}\right)$. It is then clear that $|Z|=\aleph$. If $X \in \mathcal{K}$, then there exists an ordinal $\gamma<\aleph$ such that $X=f\left(i_{\gamma}\right)$ and so we have: $Z \cap X=Z \cap f\left(i_{\gamma}\right) \subseteq\left\{j_{\alpha} \mid \alpha \leq \gamma\right\}$, where the last set is an initial segment of the well-ordered set $I$ and hence $|Z \cap X|<\kappa$. This also shows that $Z \notin \mathcal{K}$ and contradicts the maximality of $\mathcal{K}$, completing the proof.

We will say that a module $M$ is essentially $\aleph^{<}$-generated when $M$ contains an essential $\chi$-generated submodule for some cardinal $\chi<\aleph$.

Using the preceding lemma and mimicking the proof of Theorem 2.2 we obtain (in the injective case; the quasi-continuous case is similar):

Theorem 3.2. Let $R$ be $a$ ring and $E$ an injective module such that $G d(E)=\aleph$ is attained in $E$, with $\aleph$ a regular infinite cardinal. Suppose that $E$ is essentially $\aleph^{<-}$ generated and that each $\boldsymbol{N}^{<-l o c a l}$ direct summand of $E$ has a unique (as a set) injective envelope contained in $E$. Then there exists a directly split submodule $N$ of $E$ such that $G d(E / N)>G d(E)$.

Proof. We argue as in the proof of Theorem 2.2 with the following modifications. Using Lemma $3.1 I$ can be decomposed in a set $\mathcal{K} \subseteq 2^{I}$ such that $|\mathcal{K}|>\kappa,|X|=\kappa$ for each $X \in \mathcal{K}$, and $|X \cap Y|<\mathcal{N}$ for all $X, Y \in \mathcal{K}$ such that $X \neq Y$. We set $\mathcal{N}=\{A \subseteq I \| A \mid<\mathcal{N}\}$ and define $N:=\Sigma_{A \in \mathcal{N}} E_{A} \subseteq E$. Exactly as in the proof of Theorem 2.2 we see that $N$ is a directly split submodule of $E$.

Suppose now that $E_{X} \subseteq N$ for some $X \in \mathcal{K}$ and let $L_{X}$ be an essential $\mathcal{K}^{<}$-generated submodule of $E_{X}$. Then $L_{X} \subseteq E_{X} \subseteq N=\Sigma_{A \in \mathcal{N}} E_{A}$ and so there exists a set $J \subseteq \mathcal{N}$ such that $|J|<\mathcal{N}$ and $L_{X} \subseteq \Sigma_{A \in J} E_{A}$. Since $L_{X}$ is essential in $E_{X}, L_{X} \cap E_{i} \neq 0$ for each $i \in X$ and, as in the proof of Theorem 2.2 we have

$$
\oplus_{i \in X}\left(L_{X} \cap E_{i}\right) \subseteq L_{X} \subseteq \Sigma_{A \in J} E_{A} \subseteq E_{U_{A \in J A}}
$$


Since $|J|<\aleph,|A|<\aleph$ for each $A \in J$, and $\mathcal{N}$ is a regular cardinal, we have by [9, Lemma 3.2, p. 161] that $\left|\cup_{A \in J} A\right|<\mathcal{N}$. Thus we obtain

$$
\left|\cup_{A \in J} A\right|<\aleph=|X|
$$

and, as in Theorem 2.2 we have a contradiction that shows that $E_{X}$ is not contained in $N$. Therefore, $\left(E_{X}+N\right) / N \neq 0$ for each $X \in \mathcal{K}$.

Exactly as in Theorem 2.2 one shows that these modules are independent and this completes the proof.

We do not know whether Theorem 3.2 still holds without assuming $G d(E)$ regular. A more general version in which the regularity hypothesis is dropped, can be obtained if one assumes that GCH holds. If this is the case and $N$ is an infinite cardinal, then the smallest cardinal $\chi$ such that $\kappa^{x}>\aleph$ is precisely $\chi=c f(\kappa)$ (see, e.g. [9, Theorem 10.4.8, Theorem 10.4.10]). Then it follows from Tarski's lemma, with $\beta=\aleph$ that the following holds:

Lemma 3.3. Assume that $G C H$ holds and let $I$ be an infinite set, with $|I|=\kappa$ and $c f(\mathcal{K})=\chi$. Then there exists a set $\mathcal{K} \subseteq 2^{t}$ such that $|\mathcal{K}|>\mathcal{K},|X|=\chi$ for each $X \in \mathcal{K}$, and $|X \cap Y|<\chi$ for $X, Y \in \mathcal{K}, X \neq Y$.

Using Lemma 3.3 one can drop in Theorem 3.2 the hypothesis that $N$ is regular, replacing the hypotheses that every $\boldsymbol{\kappa}^{<}$-local direct summand of $E$ has a unique injective envelope in $E$ and every direct summand of $E$ has an essential $\kappa^{<}$-generated submodule, by the corresponding hypotheses on $\chi=c f(\aleph)$.

\section{REFERENCES}

1. J. Clark and Nguyen V. Dung, On the decomposition of nonsingular CS modules, Canadian Math. Bull., to appear.

2. J. Dauns and L. Fuchs, Infinite Goldie dimensions, J. Algebra 115 (1988), 297-302.

3. NGuYen V. Dung and P. F. SMITh, Hereditary CS-modules, Math. Scand. 71 (1992), 173-180.

4. Nguyen V. Dung, Dinh V. Huynh, P. F. Smith and R. Wisbauer, Extending modules (Longman, Harlow, 1994).

5. P. Erdös and A. TARSKI, On families of mutually exclusive sets, Ann. of Math. 44 (1943), 315-329.

6. A. W. GoldIE, Semi-prime rings with maximum condition, Proc. London Math. Soc. 10 (1960), 201-220.

7. J. L. Gómez Pardo and P. A. Guil Asensio, Essential embedding of cyclic modules in projectives, Trans. Amer. Math. Soc., to appear.

8. J. L. Gómez Pardo and P. A. Guil Asensio, Rings with finite essential socle, Proc. Amer. Math. Soc., 125 (1997), 971-977.

9. K. HrbaceK and T. JeCh, Introduction to set theory (Marcel Dekker, New York, 1978). 
10. S. H. Mohamed and B. J. MüLleR, Continuous and discrete modules (Cambridge University Press, Cambridge, 1990).

11. B. L. OsOFSky, A generalization of Quasi-Frobenius rings, J. Algebra 4 (1966), 373-387.

12. B. L. OsofsKy, Non-quasi-continuous quotients of finitely generated quasi-continuous modules, in Ring Theory (S. K. Jain and S. T. Rizvi, Eds., World Scientific, Singapore, 1993), 259-275.

13. P. F. Smith, Modules for which every submodule has a unique closure, in Ring Theory (S. K. Jain and S. T. Rizvi, Eds., World Scientific, Singapore, 1993), 302-313.

14. B. Stenström, Rings of Quotients (Springer-Verlag, Berlin and New York, 1975).

15. A. TARSKI, Sur la décomposition des ensembles en sous-ensembles presque disjoints, Fund. Math. 12 (1928), 188-205.

16. R. WISBAUER, Foundations of Module and Ring Theory (Gordon and Breach, Reading, 1991).

\section{Departamento de Alxebra \\ UNIVERSIDADE DE SANTIAGo \\ 15771 SaNtIago de Compostela \\ SPAIN \\ E-mail address: pardo@zmat.usc.es}

\author{
Departamento de Matematicas \\ UNIVERSIDAD DE MURCIA \\ 30100 ESPINARDO \\ Murcia \\ SPAIN \\ E-mail address: paguil@fcu.um.es
}

